\title{
Optically monitoring and controlling nanoscale topography during semiconductor etching
}

\author{
Chris Edwards, Amir Arbabi, Gabriel Popescu and Lynford L Goddard
}

\begin{abstract}
We present epi-diffraction phase microscopy (epi-DPM) as a non-destructive optical method for monitoring semiconductor fabrication processes in real time and with nanometer level sensitivity. The method uses a compact Mach-Zehnder interferometer to recover quantitative amplitude and phase maps of the field reflected by the sample. The low temporal noise of $0.6 \mathrm{~nm}$ per pixel at 8.93 frames per second enabled us to collect a three-dimensional movie showing the dynamics of wet etching and thereby accurately quantify non-uniformities in the etch rate both across the sample and over time. By displaying a gray-scale digital image on the sample with a computer projector, we performed photochemical etching to define arrays of microlenses while simultaneously monitoring their etch profiles with epi-DPM.
\end{abstract}

Light: Science \& Applications (2012) 1, e30; doi:10.1038/lsa.2012.30; published online 28 September 2012

Keywords: microscopy; nanoscale metrology; photochemical etching; quantitative phase imaging; semiconductor process monitoring

\section{INTRODUCTION}

To obtain properly functioning semiconductor devices, engineers rely on tight dimensional control during nanofabrication. In processes such as growth or etching, control of feature size, especially height, is often performed by carrying out an initial calibration run on a dummy wafer, which includes a post-process measurement to determine the growth or etching rate. After this preliminary calibration, the fabrication is run on the real wafer under the same processing conditions, followed again by a post-processing measurement check. This iterative approach has obvious drawbacks including the added time and cost of duplicate runs, variation due to system drift and lack of adaptive process control. Further, the characterization measurement often requires destruction of the sample. It is clear that accurate, non-destructive, real-time in situ monitoring is highly desirable as it enables feedback and fine adjustment of the processing conditions.

Optical characterization methods fulfill the need for nondestructive testing. Thus, point-measurement techniques such as spectroscopic ellipsometry, ${ }^{1-4}$ phase-sensitive ellipsometry, ${ }^{5}$ laser reflectometry, ${ }^{6,7}$ multi-beam interferometry, ${ }^{8,9}$ and emission spectroscopy ${ }^{10,11}$ have been successfully implemented. Typically, the structure height is measured at a single point or region of interest and information across the wafer is inferred assuming the process is uniform. This is adequate for most planar processes, but it is well known that the growth and etch rates in high aspect ratio structures, e.g., at the bottom of a trench, depend on the width of the opening. Consequently, an imaging rather than a single-point method is preferred. Along these lines, imaging ellipsometry has recently been used to characterize graphene on flat surfaces ${ }^{12}$ and to monitor the removal of large area biomolecules during plasma etching. ${ }^{13}$ The sensitivity of these single-point or imaging methods can be a few nanometers; however, the measurements are typically hindered by vibrations and drift in the sample. The ability to maintain nanoscale accuracy in noisy environments is extremely difficult and represents the main challenge in developing real-time monitoring of dynamic fabrication processes such as wet etching.

During the past decade or so, quantitative phase imaging, i.e., mapping the path length shift created by a sample at each point in the image, has received increasing scientific interest in life sciences. ${ }^{14}$ The phase images from quantitative phase imaging provide information at the nanoscale about the structure and dynamics of the specimen under investigation. In particular, diffraction phase microscopy $(D P M)^{15}$ is a stable quantitative phase imaging method that has been used successfully to study nanoscale fluctuations of cell membranes. ${ }^{15-18}$

\section{MATERIALS AND METHODS}

Here, we present a new optical approach that exploits the concept of DPM to perform real-time quantitative topographic measurements of nanoscale dynamics. Our method, referred to as epi-diffraction phase microscopy (epi-DPM), operates in reflection to accommodate opaque samples, and renders topography information with $2.8 \mathrm{~nm}$ spatial (i.e., point to point) and $0.6 \mathrm{~nm}$ temporal (frame to frame) sensitivity. The nanoscale topographic images are obtained from a single camera exposure, such that the acquisition rate is only limited by the camera frame rate. Furthermore, epi-DPM is completely non-invasive and allows for the continuous in-situ monitoring of a 
fabrication process in real-time through a transparent window on the tool, without compromising the nanoscale topographic accuracy. In particular, we present dynamic imaging data of semiconductor structures while being etched; epi-DPM reveals quantitatively the etch rate at each point in the image and each moment in time. We found this rate to vary significantly versus position and time with typical values in the range of $0-8 \mathrm{~nm} \mathrm{~s}^{-1}$. Finally, we show that photochemical etching can be used in combination with epi-DPM imaging to controllably vary the etch rate across the sample and thereby fabricate 'gray-scale' structures, e.g., microlenses. Thus, the use of a computer projector to display different optical masks combined with real-time feedback from epi-DPM allows for the fabrication of structures with arbitrary topographic profiles, without the need for iterative or multistep etching or expensive gray-scale lithography masks.

The experimental setup is shown in Figure 1. Light at $532 \mathrm{~nm}$ from a frequency-doubled Nd:YAG laser is used as the epi-illumination source. The laser beam is coupled into a single-mode fiber and then collimated, which ensures full spatial coherence of the output field. This collimated light then enters the back port of an inverted microscope and is projected to the sample plane by a collector lens and the objective. Thus, the sample is illuminated by a collimated beam. The light reflected from the sample of interest is collected through the same objective, passed through a beam splitter and tube lens, and directed toward a side output port of the microscope.

To quantify the phase shift distribution across the field of view with high stability, we constructed a compact Mach-Zehnder interferometer, as follows. A diffraction grating was placed at the image plane of the microscope such that multiple copies of the image are generated at different angles. Note that at the image plane, we obtain a magnified replica of the field reflected by the sample. The diffraction grating had 300 grooves $\mathrm{mm}^{-1}$, blazed at an angle to maximize the power in the first diffraction order. Lens $\mathrm{L}_{3}$ is used to generate the Fourier transform of the image field at its back focal plane. At this Fourier plane, the first order beam is filtered down using a $10 \mu \mathrm{m}$ diameter pinhole, such that after passing through the second lens $\left(\mathrm{L}_{4}\right)$, this field approaches a plane wave and, thus, can be used as the reference of our interferometer. The original image is carried by the zeroth order, which is combined with the reference field at the charge-coupled device camera plane and creates an interferogram that has the following irradiance distribution,

$$
I(x, y)=I_{1}+I_{0}(x, y)+2 \sqrt{I_{1} I_{0}(x, y)} \cos [\beta x+\varphi(x, y)]
$$

where $I_{0}$ is the irradiance of the zeroth diffraction order, $I_{1}$ is the irradiance of the first diffraction order after passing the pinhole, $\beta$ is the spatial modulation frequency provided by the grating and $\varphi$ is the phase of interest. From this interferogram, the quantitative phase image is obtained via a spatial Hilbert transform. ${ }^{19}$ The phase image is then unwrapped using the Goldstein's branch cut algorithm ${ }^{20}$ and converted into a height image.

\section{RESULTS AND DISCUSSION}

The strength of epi-DPM is that it provides accurate topographic height maps relative to other points in the image and therefore can be made insensitive to any common mode motion of the sample. To characterize the spatial and temporal path length noise of our epi-DPM method, we repeatedly imaged a plain, unprocessed $n+$ GaAs wafer. Thus, we acquired a time-lapse series of images containing 256 frames at 8.93 frames s $^{-1}$. We used a $10 \times$ objective (numerical aperture $=0.25$ ), which provides a diffraction limited lateral resolution of $1 \mu \mathrm{m}$. Note that the lateral resolution could be improved considerably by using shorter wavelength light (e.g. deep ultraviolet) or a higher numerical aperture objective. The field of view is approximately $160 \mu \mathrm{m} \times 120 \mu \mathrm{m}$. After recovering the height images, a quadratic fit of each individual image was subtracted off. The linear portion of the fit corrects for the tilt of the sample and the angle of interference in the camera plane, while the quadratic portion corrects for the quadratic phase front of the beam across the field of view. After correction, the spatial standard deviation of the image at each time frame, $\sigma_{\mathrm{xy}}(t)$, was computed. The median value was $11.2 \mathrm{~nm}$.

It is well known that the speckles associated with the laser light contribute significantly to the spatial phase noise floor. To further lower the spatial noise, we inserted a rotating diffuser in the illumination path (see the supplementary information section for details). This method essentially averages out the effects of speckles. ${ }^{21,22}$ It also dithers the image, which reduces the quantization error. The

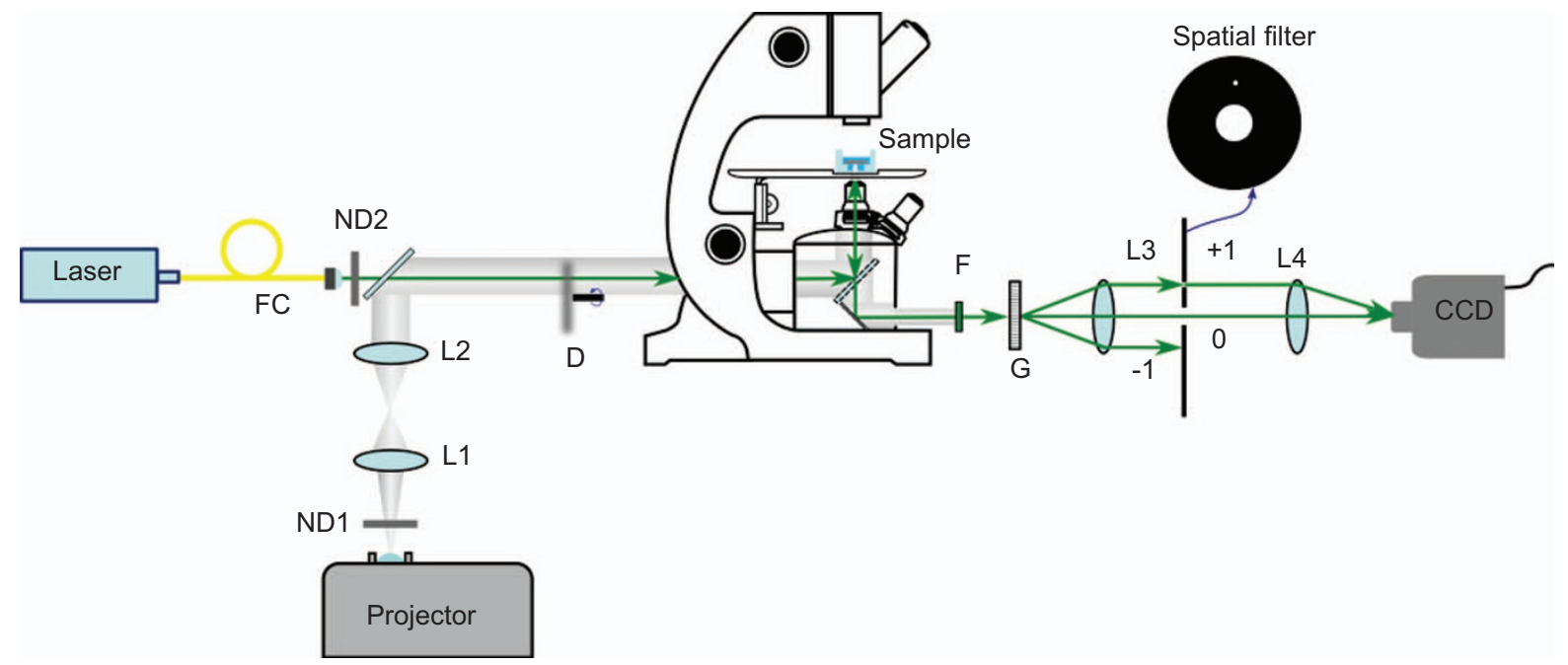

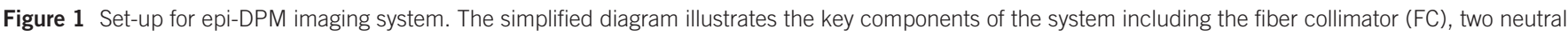

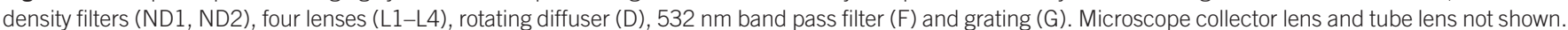
epi-DPM, epi-diffraction phase microscopy. 
resulting epi-DPM images revealed that the spatial noise floor dropped to $10.4 \mathrm{~nm}$ after adding the diffuser.

Imperfections in optical components lead to non-uniformities across the field of view in the phase of the light that impinges on the sample. Since this type of phase error is reproducible, i.e., it is a characteristic of the instrument, it can be largely removed through proper system calibration. Thus, we collected a second time lapse series of images both with and without the diffuser at a different spatial location, separated by approximately $0.5 \mathrm{~mm}$, i.e., several fields of view away. Each series at the first location was averaged in time and used as the calibration image. There is a different calibration image for operation with and without the diffuser. The respective calibration image was subtracted from each image at the second location. The resulting spatial noise was $4.6 \mathrm{~nm}$ without the diffuser and $2.8 \mathrm{~nm}$ with the diffuser. The value of $2.8 \mathrm{~nm}$ represents the spatial sensitivity of our current instrument and the final sensitivity to lateral topography changes within the field of view. Remarkably, due to the common path interferometric geometry employed, the temporal sensitivity of epiDPM is significantly better than the spatial sensitivity. The temporal standard deviation of the height at each point in the image, $\sigma_{\mathrm{t}}(x, y)$, is shown in Figure 2a and 2b. A histogram of $\sigma_{t}(x, y)$ is shown in each inset. We determined the temporal sensitivity as the median
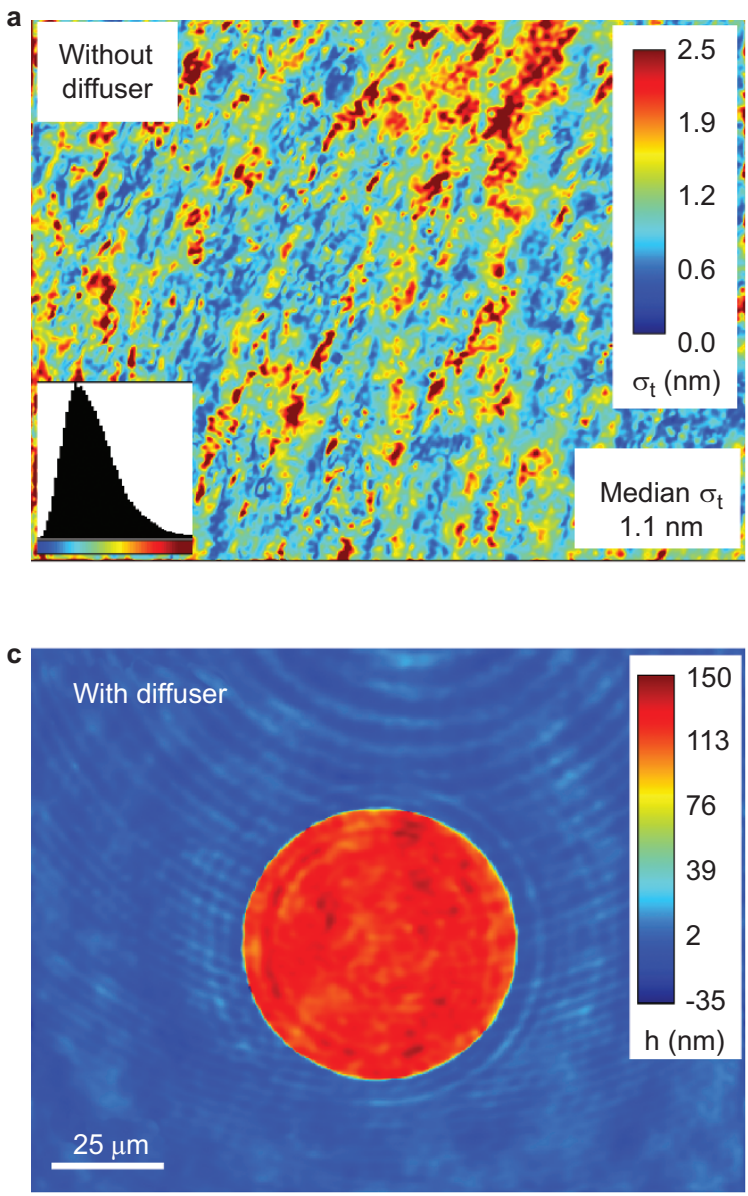

of this histogram to be $1.1 \mathrm{~nm}$ without the diffuser and $0.6 \mathrm{~nm}$ with the diffuser.

In the Supplementary Information, we analyze the power spectral density of the noise (Supplementary Figure S1). The spectrum reveals low frequency noise as well as white noise. The diffuser significantly reduces the low frequency noise as well as its spectral width. Given the noise spectrum, it may be possible to reduce the noise floor by one to two orders of magnitude by performing lock-in detection both temporally and spatially (see Chap. 8 in Ref. 14).

Curvature removal and background subtraction are performed on all measurement images collected with epi-DPM. However, given the low temporal noise, only one image is collected for calibration instead of the 256 frames for noise characterization.

To characterize the accuracy of epi-DPM, images of micropillars fabricated by wet etching of an $n+$ GaAs wafer were collected. Photolithography and developing were done using a standard SPR 511A photoresist recipe described in the supplementary information section. Figure $2 \mathrm{c}$ shows the epi-DPM image of a micropillar with the diffuser. The positions of the pillar and etched region were extracted from the histogram of the epi-DPM image as shown in Figure $2 \mathrm{~d}$ by fitting each histogram peak to a separate Gaussian distribution (see Supplementary Figure S2 for the corresponding image and

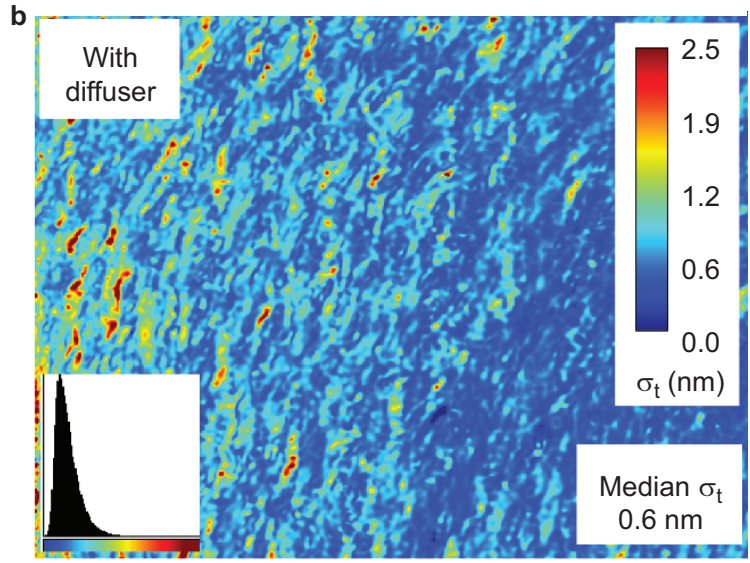

d

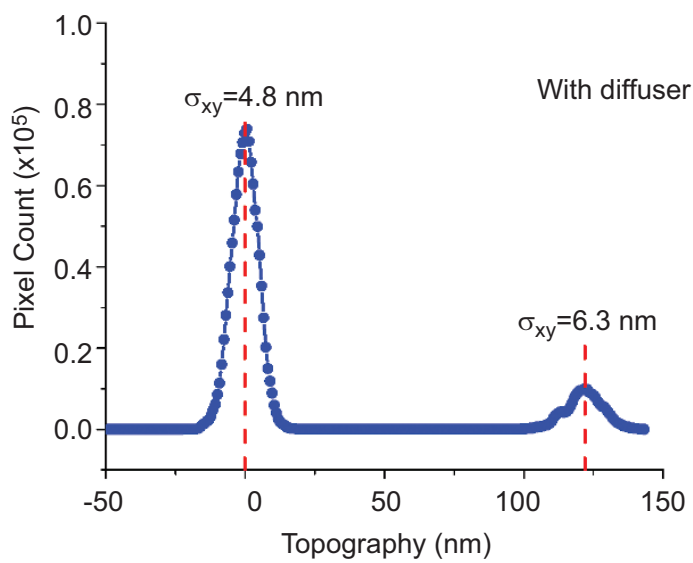

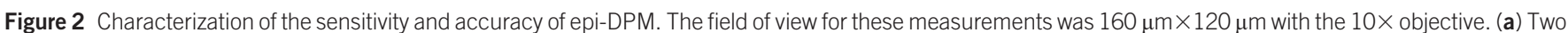

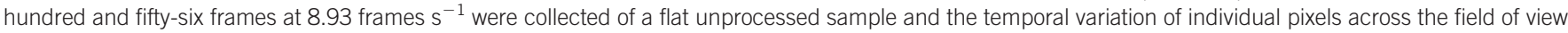

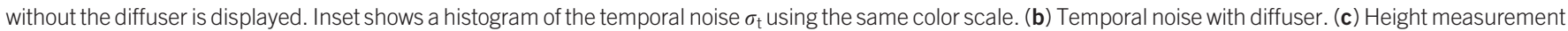

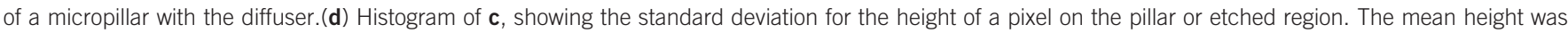

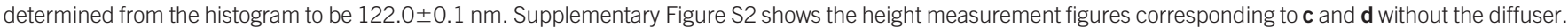
epi-DPM, epi-diffraction phase microscopy. 
histogram without the diffuser). Although the spatial noise from an individual pixel is several nanometers, the standard error in the mean position is $0.1 \mathrm{~nm}$ after averaging and assuming that pixels more than a diffraction spot diameter apart are independent. Thus, the mean height was $122.0 \pm 0.1 \mathrm{~nm}$. This agrees very well with our measurements taken by the Tencor Alpha-Step 200 surface profiler and the Hitachi S-4800 scanning electron microscope, which gave values between 120 and $130 \mathrm{~nm}$ for the various micropillars. A small degree of non-uniformity among the micropillar heights was observed, which is typical of the fabrication process. For epi-DPM, the spatial standard deviation in height was $6.3 \mathrm{~nm}$ for the unetched circular pillar and $4.8 \mathrm{~nm}$ for the etched region. The background subtraction calibration procedure reduced the spatial noise in both regions, but was not as effective as on the flat sample. Because the noise contribution from laser speckle is due to interference, it cannot be properly removed by subtracting the calibration image which contains the interference pattern for a flat surface. However, the portion of the spatial noise due to non-uniformities in the input phase can still be removed. For these reasons, we believe that the spatial noise is lower on the etched region but higher than the flat sample. The larger flat
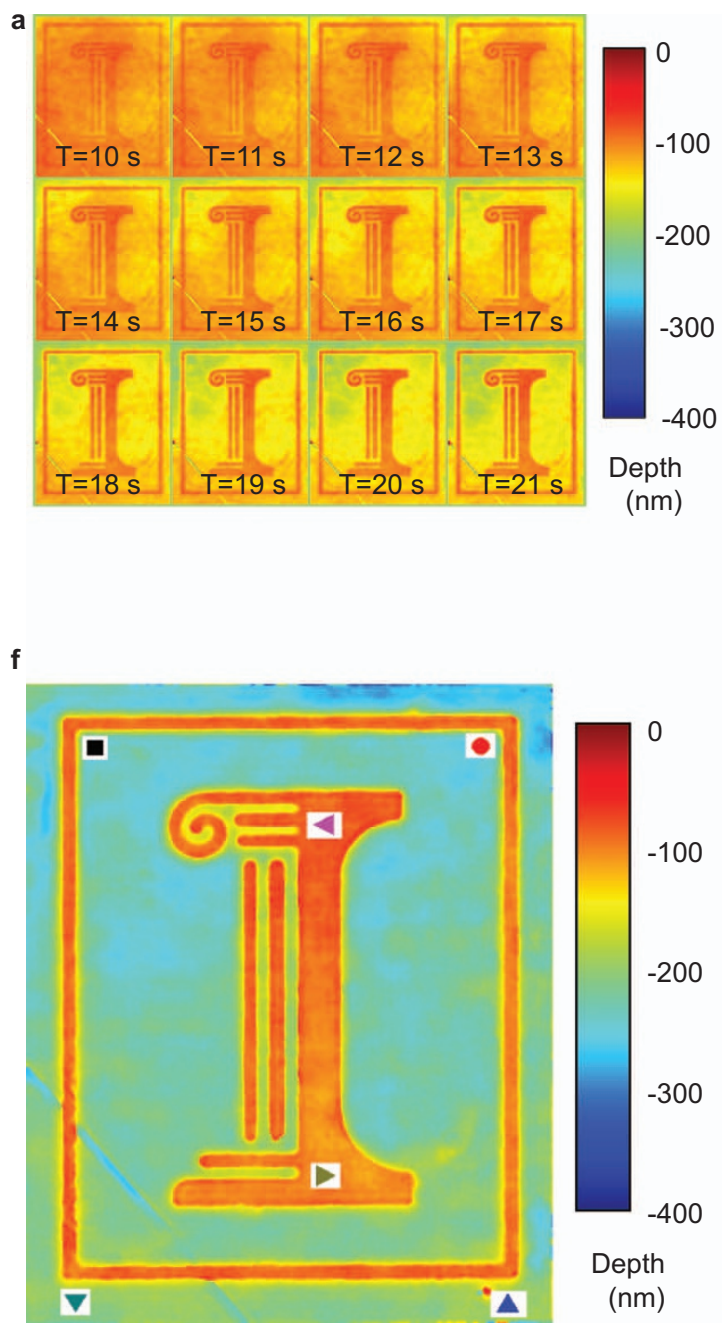

area enables better subtraction. Also, the wet etching process tends to smoothen the semiconductor surface.

These sensitivity and accuracy results underscore the capability of epi-DPM to monitor dynamic topographic changes at the nanoscale. To exploit this feature, we imaged the topography of an $n+$ GaAs wafer while being etched with a solution of $\mathrm{H}_{3} \mathrm{PO}_{4}: \mathrm{H}_{2} \mathrm{O}_{2}: \mathrm{H}_{2} \mathrm{O}$ (see Supplementary Information for details on the processing steps). ${ }^{23}$ We acquired 400 frames at 8.93 frames $\mathrm{s}^{-1}$, for a total of $44.8 \mathrm{~s}$. Our epi-DPM movie (Supplementary Movie) revealed that it took approximately $10 \mathrm{~s}$ for the etchant to diffuse into the field of view and begin etching. The montage in Figure $3 \mathrm{a}$ indicates the spatial inhomogeneity and the time evolution of the etching process. The quantitative information provided by epi-DPM allows us to measure etch rates in a spatially and temporally resolved manner. Thus, Figure $3 \mathrm{~b}-3 \mathrm{~d}$ show the etch rate maps across the structure at different moments during the process, while Figure $3 \mathrm{e}$ displays the overall (mean) etch rate. The resolution of the etch rate images shown in Figure $3 \mathrm{~b}-3 \mathrm{~d}$ is about $0.085 \mathrm{~nm} \mathrm{~s}^{-1}$ given the temporal noise of $0.6 \mathrm{~nm}$ per pixel, the addition of noise in quadrature and the 10-s time interval used to compute the derivative of the height images such as

b
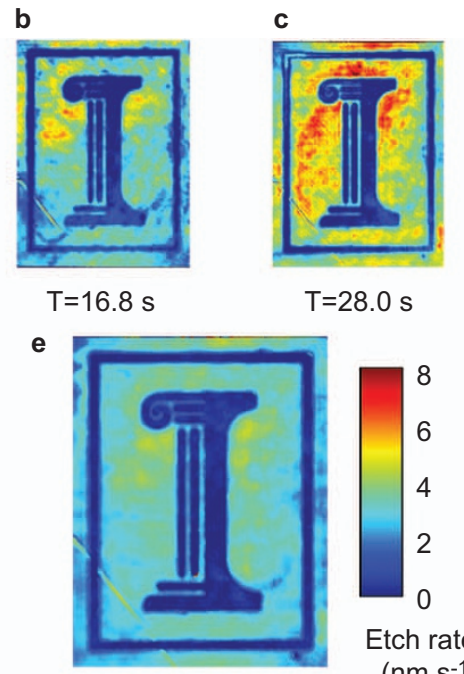

Averaged for $\mathrm{T}=0-44.8 \mathrm{~s}$

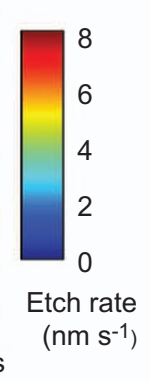

g

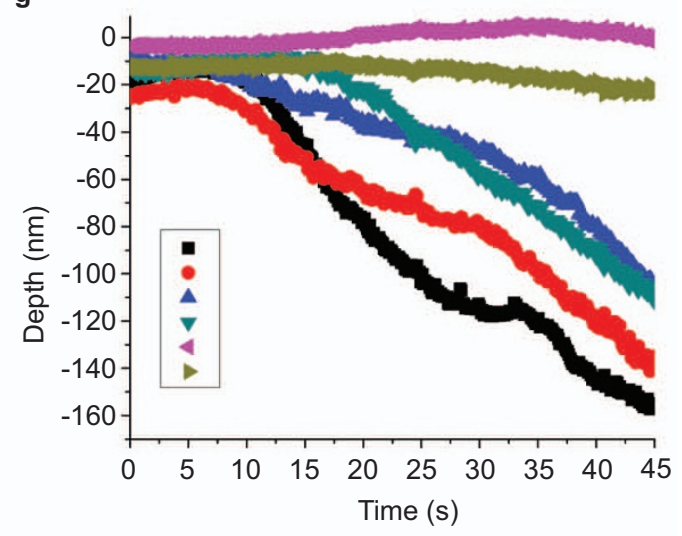

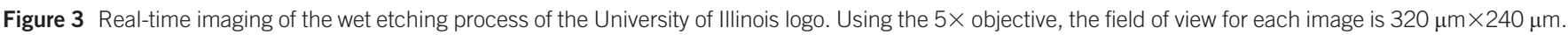

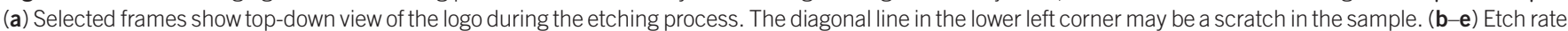

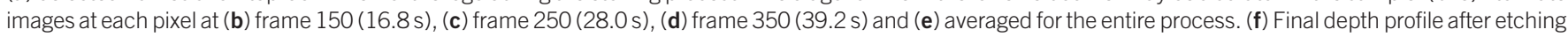
was completed. (g) Plot of etching depths over time to show variation of depths and etch rates at points marked in $\mathbf{f}$. 
in Figure 3a. Figure $3 \mathrm{f}$ shows the final etched structure, while Figure $3 \mathrm{~g}$ illustrates the time-resolved etch depth profile at discrete points across the sample, as indicated by the markers in Figure $3 \mathrm{f}$. These data reveal subtle details regarding the etching process. In particular, we found that the etching process is considerably inhomogeneous, i.e., the etch rate can easily vary by a factor of 5-10 at unmasked points separated by only a few microns in space at a fixed moment in time or at times separated by only a few seconds at a fixed point in space. This unexpected inhomogeneity is most likely due to fluctuations in the concentration of acid in the solution. The ability to quantify these inhomogeneities in real time may provide the means to correct the fabrication on-the-fly, for example, by perfusing the etching chamber with an adjustable concentration of etchant.

In combination with epi-DPM, photochemical etching may offer an efficient means to fabricate structures with precisely controllable topography. The method is well suited for fabricating complex grayscale structures which are typically very difficult or expensive to produce using standard photolithographic techniques. As an example, we used epi-DPM to image microlens structures fabricated by photochemical etching. Photochemical etching is a process by which light absorption in a semiconductor increases the concentration of minority carriers which then diffuse to the surface and thereby increase the rate of dissolution and corrosion of the semiconductor in an oxidizing solution. ${ }^{24-28}$ The etch rate is limited by the supply of minority carriers to the surface; therefore, the illumination accelerates the etching process. ${ }^{24-28}$ We added a common light projector (EPSON Powerlite S5 EMP-S5) in the illumination path, such that gray-scale images delivered by a computer can be mapped onto the sample, as shown in Figure 1. To demonstrate the ability of epi-DPM to monitor gray-scale structures produced by photochemical etching, we fabricated individual microlenses by simply using a digital image as an optical mask. Figure 4a shows the mask delivered by the projector to the sample plane and Figure $4 \mathrm{~b}$ is the corresponding epi-DPM image of the resulting fabricated microlens. The samples were plain, unprocessed $n+$ GaAs wafers and the etch time was calibrated to 2 min for a lens height of $500 \mathrm{~nm}$ and a diameter of $100 \mu \mathrm{m}$. The measured height and diameter for the resulting microlens depicted in Figure $4 \mathrm{~b}$ were $590 \mathrm{~nm}$ and $100 \mu \mathrm{m}$, respectively. Both the projector and the imaging laser etch the sample. The laser etches uniformly, while the projector etches the pattern. The laser power was minimized to reduce unwanted etching. At the sample plane, the laser power density was $0.64 \mathrm{~mW} \mathrm{~cm}^{-2}$, while the projector delivered $8.9 \mathrm{~mW} \mathrm{~cm}^{-2}$. The a

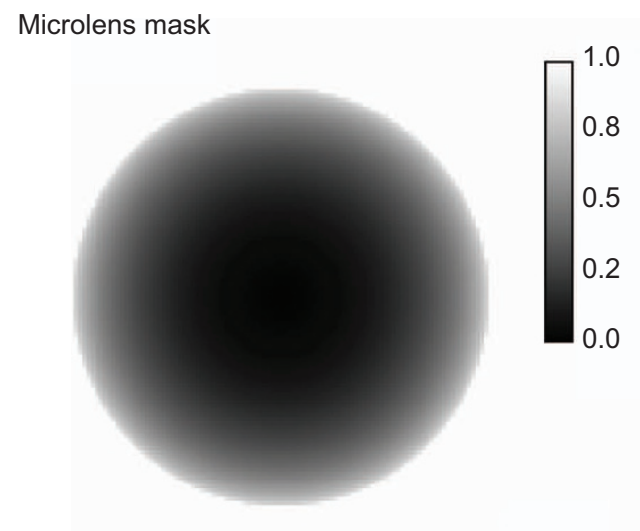

$20 \mu \mathrm{m}$

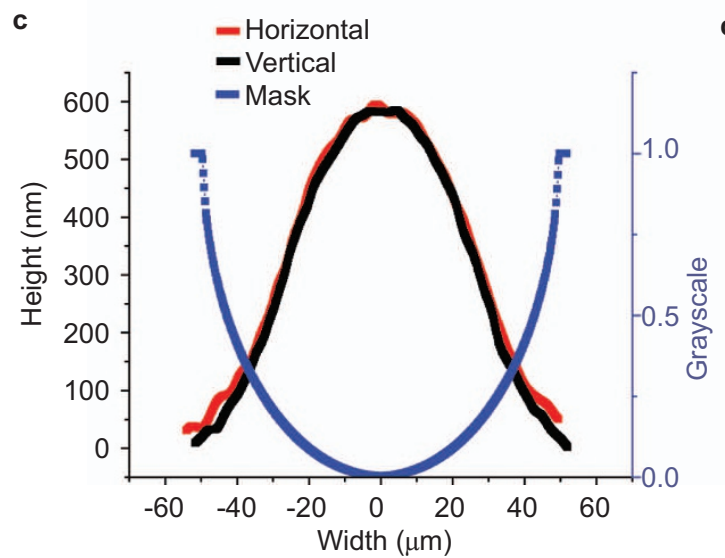

b

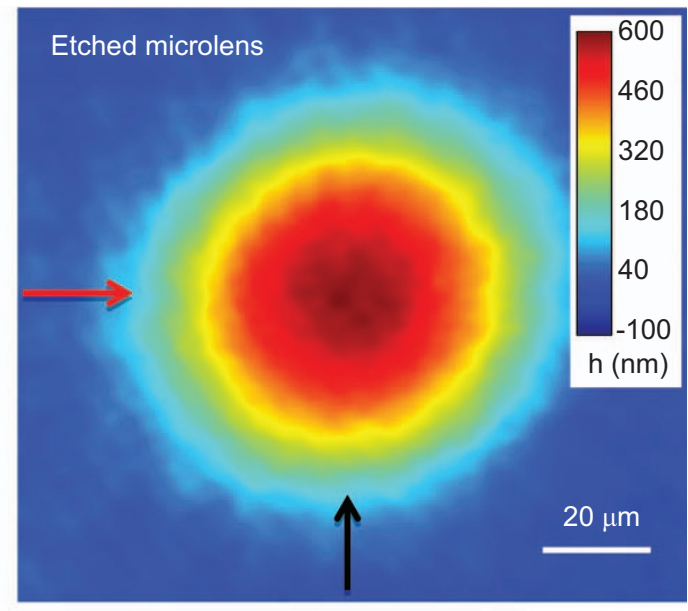

d

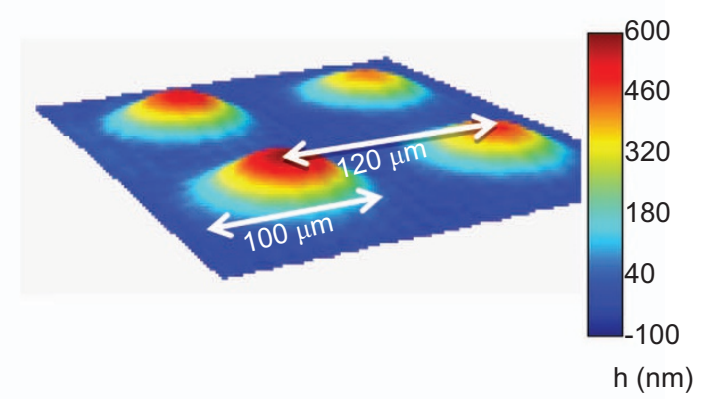

Figure 4 Computer projector based photo-chemical etching of microlenses. (a) Digital image used to etch microlenses using only light from a projector and an isotropic wet etch solution. (b) Image of an etched microlens displaying top-down view. (c) Projector intensity profile and comparison of horizontal and vertical cuts of $\mathbf{b}$ to illustrate symmetry. (d) Microlens array displaying height, lens diameter and pitch. Heights were between 425 and $590 \mathrm{~nm}$, the diameters ranged from 95 to $105 \mu \mathrm{m}$ and the pitch varied from 120 to $125 \mu \mathrm{m}$. The field of view for images $\mathbf{b}$ and $\mathbf{d}$ was $320 \mu \mathrm{m} \times 240 \mu \mathrm{m}$ using the $5 \times$ objective. 
power density of the laser under normal imaging conditions without attenuation was $57 \mathrm{~mW} \mathrm{~cm}^{-2}$.

The profiles through the mask and the microlens (Figure 4c) indicate that the photochemical etching process is nonlinear, i.e., the local lens height is not simply proportional to the light irradiance delivered at that point. However, epi-DPM can measure precisely this nonlinear relationship and, in turn, provide a calibration curve for etching structures with prescribed profiles. In addition, imaging with epi-DPM can render the feedback necessary to fine-tune the etching process in real time, by controlling the projector light intensity across the field of view.

We also fabricated $2 \times 2$ arrays of microlenses, as illustrated in Figure $4 \mathrm{~d}$. The optical mask in this case contained five $2 \times 2$ arrays of gray-scale lenses with $100 \mu \mathrm{m}$ diameters and $120 \mu \mathrm{m}$ pitch. The resulting heights ranged from about 425 to $590 \mathrm{~nm}$ across the array, the diameters from 95 to $105 \mu \mathrm{m}$ and the pitch ranged from 120 to $125 \mu \mathrm{m}$. The surface profiler verified that the diameters and pitch were within $2 \mu \mathrm{m}$ and the heights were within $10 \mathrm{~nm}$ of the values measured by epi-DPM. Better height uniformity across the array can be obtained by implementing adaptive control of the projector's mask pattern.

\section{CONCLUSIONS}

In summary, we have shown how epi-DPM can be used to accurately monitor the dynamics of semiconductor fabrication processes in real time. The method has an excellent temporal stability of $0.6 \mathrm{~nm}$ per pixel at 8.93 frames $s^{-1}$. This enabled us to accurately determine spatial and temporal variations in the etch rate with a resolution of $0.085 \mathrm{~nm} \mathrm{~s}^{-1}$ for a 10 -s time interval. In conjunction with epi-DPM, we performed photochemical etching to precisely define nanoscale topographical features such as a microlens array.

We envision that using epi-DPM to image through transparent viewing windows of semiconductor manufacturing tools will enable engineers to better monitor and control the performance properties of their fabricated devices. To watch an entire wafer die during processing, the field of view can be increased by using a higher resolution camera. At the time of writing, there are commercially available 80 megapixel cameras (2.6" diagonal charge-coupled device). Also, higher numerical aperture $10 \times$ objectives are currently available (e.g., numerical aperture $=0.5$ ). This combination of camera and objective would enable a $1.0 \mathrm{~mm} \times 0.76 \mathrm{~mm}$ field of view with $500 \mathrm{~nm}$ lateral resolution. Further, using a shorter wavelength light source (e.g., deep ultraviolet) would significantly reduce the diffraction limited lateral resolution and could enable in situ monitoring of some of today's smallest devices.

\section{AUTHOR CONTRIBUTIONS}

All authors contributed in conceiving and designing the experiments. GP conceived epi-DPM. CE and AA performed the experiments. CE, LG and AA wrote the data analysis software. All authors interpreted the data, discussed the results, and wrote the paper.

\section{COMPETING FINANCIAL INTEREST}

G Popescu is founder of Phi Optics, Inc., a company developing quantitative phase imaging technology for materials and life science applications.

\section{ACKNOWLEDGMENTS}

The authors thank Hoa Pham for assistance with the power spectral density calculations and Brian Cunningham, Xiuling Li, Logan Liu and Daniel
Wasserman for helpful discussions. This work is supported by NSF CBET1040462 MRI award.

1 Maynard HL, Layadi N, Lee JTC. Plasma etching of submicron devices: in situ monitoring and control by multi-wavelength ellipsometry. Thin Solid Films 1998; 313/314: 398-405.

2 Langereis E, Heil SBS, Knoops HCM, Keuning W, van de Sanden MCM et al. In situ spectroscopic ellipsometry as a versatile tool for studying atomic layer deposition. J Phys D: Appl Phys 2009; 42: 073001.

3 Losurdo M, Bergmair M, Bruno G, Cattelan D, Cobet C et al. Spectroscopic ellipsometry and polarimetry for materials and systems analysis at the nanometer scale: state-ofthe-art, potential, and perspectives. J Nanopart Res 2009; 11: 1521-1554.

4 Aspnes DE, Bagley BG. Method for optical monitoring in materials fabrication. US Patent 4,332,833, 1982

5 Hall R. Phase sensitive optical monitor for thin film deposition. US Patent 4,906,844, 1990

6 Rebey A, Boufaden T, Jani BE. In situ optical monitoring of the decomposition of GaN thin films. J Crystal Growth 1999; 203: 12-17.

7 Song YM, Chang KS, Na BH, Yu JS, Lee YT. Precise etch-depth control of microlensintegrated intracavity vertical-cavity surface-emitting lasers by in-situ lase reflectometry and reflectivity modeling. Thin Solid Films 2009; 517: 5773-5778.

8 Tosaka H, Minami K, Esashi M. Optical in situ monitoring of silicon diaphragm thickness during wet etching. J Micromech Microeng 1995; 5: 41-46.

9 Burrow GM, Gaylord TK. Multi-beam interference advances and applications: nanoelectronics, photonic crystals, metamaterials, subwavelength structures, optical trapping, and biomedical structures. Micromachines 2011; 2: 221-257.

10 Mackus AJM, Heil SBS, Langereis E, Knoops HCM, van de Sanden MCM et al. Optical emission spectroscopy as a tool for studying, optimizing, and monitoring plasmaassisted atomic layer deposition processes. J Vac Sci Technol A 2010; 28: 77-87.

11 Guinn KV, McNevin SC. Process for device fabrication in which the plasma etch is controlled by monitoring optical emission. US Patent 5,877,032, 1999

12 Wurstbauer U, Röling C, Wurstbauer U, Wegscheider W, Vaupel M et al. Imaging ellipsometry of graphene. App/ Phys Lett 2010; 97: 231901.

13 Rauscher H, Stapelmann K, Kylian O, Denis B, Rossi F. Monitoring plasma etching of biomolecules by imaging ellipsometry. Vaccum 2009; 84: 75-78.

14 Popescu G. Quantitative phase imaging of cells and tissues. New York: McGraw-Hill, 2011.

15 Popescu G, Ikeda T, Dasari RR, Feld MS. Diffraction phase microscopy for quantifying cell structure and dynamics. Opt Lett 2006; 31: 775-777.

16 Park YK, Best CA, Auth T, Gov N, Safran SA et al. Metabolic remodeling of the human red blood cell membrane. Proc Natl Acad Sci USA 2010; 107: 1289-1294.

17 Park YK, Best CA, Badizadegan K, Dasari RR, Feld MS et al. Measurement of red blood cell mechanics during morphological changes. Proc Natl Acad Sci USA 2010; 107: 6731-6736.

18 Park YK, Diez-Silva M, Popescu G, Lykotrafitis G, Choi W et al. Refractive index maps and membrane dynamics of human blood cells parasitized by Plasmodium falciparum. Proc Natl Acad Sci USA 2008; 105: 13730-13735.

19 Ikeda T, Popescu G, Dasari RR, Feld MS. Hilbert phase microscopy for investigating fast dynamics in transparent systems. Opt Lett 2005; 30: 1165-1167.

20 Ghiglia DC, Pritt MD. Two-dimensional phase unwrapping: theory, algorithms, and software. New York: Wiley, 1998.

21 Khmaladze A, Kim M, Lo CM. Phase imaging of cells by simultaneous dual-wavelength reflection digital holography. Opt Exp 2008; 16: 10900-10911.

22 Kubota S, Goodman JW. Very efficient speckle contrast reduction realized by moving diffuser device. Appl Opt 2010; 49: 4385-4391.

23 Williams KR, Gupta K, Wasilik M. Etch rates for micromachining processes-part II. J Micromechan Syst 2003; 12: 761-778.

24 Houle FA. Photochemical etching of silicon: the influence of photogenerated charge carriers. Phys Rev B 1989; 39: 10120-10132.

25 Look DC. Semiconductors and semimetals. New York: Academic, 1983.

26 Noguchi N, Suemune I. Luminescent porous silicon synthesized by visible light irradiation. Appl Phys Lett 1993; 62: 1429-1431.

27 Ouyang H, Deng Y, Knox WH, Fauchet PM. Photochemical etching of silicon by two photon absorption. Phys Status Solidi 2007; 204: 1255-1259.

28 Trichas E, Kayambaki M, Illiopoulos E, Pelekanos NT, Savvidis PG. Resonantly enhanced selective photochemical etching of GaN. Appl Phys Lett 2009; 94 173505. creativecommons.org/licenses/by-nc-nd/3.0 


\section{ERRATUM}

\section{Optically monitoring and controlling nanoscale topography during semiconductor etching}

Chris Edwards, Amir Arbabi, Gabriel Popescu and Lynford L Goddard

Light: Science \& Applications (2012) 1, e44; doi:10.1038/lsa.2012.44; published online 19 October 2012

Correction to: Light: Science \& Applications (2012) 1, e30; doi:10.1038/lsa.2012.30

This article, published on 28 September, should have been published with an accompanying supplementary information video file online.

This supplementary information file has now been published online; we apologize for the omission. 